\title{
Erratum to: Injection of Aß1-40 into hippocampus induced cognitive lesion associated with neuronal apoptosis and multiple gene expressions in the tree shrew
}

\author{
Na Lin ${ }^{1} \cdot$ Liu-Lin Xiong ${ }^{2} \cdot$ Rong-ping Zhang ${ }^{1} \cdot$ Hong Zheng ${ }^{1} \cdot$ Lei Wang $^{3}$ • \\ Zhong-Yi Qian $^{1} \cdot$ Piao Zhang ${ }^{1} \cdot$ Zhi-wei Chen $^{4} \cdot$ Fa-Bao Gao ${ }^{3}$ Ting-Hua Wang ${ }^{1,2,4}$
}

Published online: 7 April 2016

(C) Springer Science+Business Media New York 2016

\section{Erratum to: Apoptosis}

DOI 10.1007/s10495-016-1227-4

The original version of this article unfortunately contained a mistake. Author's would like to include the missing Acknowledgments through this erratum.
Acknowledgments This research was supported by a grant from National Key Technology Research and Development Program of the Ministry of Science and Technology of China. (CN) (No. 2014BAI01B00).

$\mathrm{Na}$ Lin, Liu-Lin Xiong, Fa-Bao Gao and Ting-Hua Wang have contributed equally to this work.

The online version of the original article can be found under doi:10.1007/s10495-016-1227-4.

Ting-Hua Wang

tinghua_neuron@263.net

1 Institute of Neuroscience, Center of Experimental Animals, Kunming Medical University, Kunming 650031, China

2 Department of Anesthesiology, Institute of Neurological Disease, Translational Neuroscience Center, West China Hospital, Sichuan University, Chengdu 610041, China

3 Molecular Imaging Laboratory, Department of Radiology, West China Hospital, Sichuan University, Chengdu 610041, China

4 Key Laboratory of National Physical Fitness and Altitude Training Adaptation in Yunnan Province, Institute of Physical Education, Yunnan Normal University, Kunming 650500, China 